\title{
Anyon in External Electromagnetic Field: Hamiltonian and Langrangian Formulations
}

\author{
by
}

\author{
M. Chaichian ${ }^{1,2}$, R. Gonzalez Felipe ${ }^{1, \text { 冈, D.Louis Martinez }}{ }^{3, *, \text { | }}$
}

\begin{abstract}
${ }^{1}$ High Energy Physics Laboratory, Physics Department, P.O. Box 9 (Siltavuorenpenger $20 \mathrm{C}$ ), SF-00014, University of Helsinki, Finland

${ }^{2}$ Research Institute for High Energy Physics, P.O. Box 9 (Siltavuorenpenger 20 C), SF-00014, University of Helsinki, Finland

${ }^{3}$ Research Institute for Theretical Physics, P.O. Box 9 (Siltavuorenpenger 20C), SF-00014, University of Helsinki, Finland
\end{abstract}

\begin{abstract}
We propose a simple model for a free relativistic particle of fractional spin in $2+1$ dimensions which satisfies all the necessary conditions. The canonical quantization of the system leads to the description of one-particle states of the Poincaré group with arbitrary spin. Using the Hamiltonian formulation with the set of constraints, we introduce the electromagnetic interaction of a charged anyon and obtain the Lagrangian. The Casimir operator of the extended algebra, which is the first-class constraint, is obtained and gives the equation of motion of the anyon. In particular, from the latter it follows that the gyromagnetic ratio for a charged anyon is two due to the parallelness of spin and momentum of the particle in $2+1$ dimensions. The canonical quantization is also considered in this case.
\end{abstract}

* On leave of absence from Grupo de Fisica Teórica, ICIMAF, Academia de Ciencias de Cuba, Calle E No. 309, Vedado, Habana 4, Cuba.

${ }^{\dagger}$ Present address: Department of Physics, Univ. of Manitoba, Winnipeg MB R3T 2N2, Canada. 


\section{Introduction}

The existence of anyons or particles with arbitrary spin and statistics in $2+1$ dimensions [1] has been attracting a great deal of attention due to the applications to different planar physical phenomena such as the fractional quantum Hall effect and possibly, high-Tc superconductivity and to the description of physical processes in the presence of cosmic strings. Several field-theoretic models have been proposed, in which anyons appear as topological solitons $[2,3]$ or electrically charged vortices [4,5]. In another approach, point particles, described by scalar or spinor fields, are coupled minimally to a $U(1)$ gauge field, the so-called statistical gauge field, whose dynamics is governed by the Chern-Simons action [6]. However, none of the above models gives a description for a free particle with arbitrary spin.

In Refs. [7,8] the field equations for a free particle with fractional spin were proposed and it was shown that their solutions realize the one-particle states as the appropriate induced representation of the Poincaré group in $2+1$ dimensions. Besides, in [7] the corresponding classical action for the fields was constructed by analogy with the action of the massive vector field. Ref. [9] dealt with the same problem, but starting from the description of the classical action for a relativistic particle with fixed mass and fixed arbitrary spin. There the set of Hamiltonian constraints was found and two different schemes of the canonical quantization of the model were considered. However, no interaction of anyons with the electromagnetic field has been considered in the above mentioned works.

In Ref. [10] based on heuristical arguments an equation for anyon in a constant magnetic field was assumed for the description of relativistic fractional quantum Hall effect. In Ref. [11], using the approach of coupling fermions or bosons to a statistical Chern-Simons field, it was shown that one-anyon states acquire an induced magnetic moment consistent with a value of $g=2$ for the gyromagnetic ratio. In a recent paper [12] the electromagnetic interaction of anyons has been considered on the basis of classical analogy with the behaviour of spin in an electromagnetic field and intuitive arguments.

In this letter we propose Hamiltonian and Lagrangian descriptions for both a free and an interacting (with electromagnetic field) relativistic particles with fractional spin. Our derivations are based on the imposed constraints and the algebraic properties of the system, namely on the invariants, Casimir operators, of the corresponding extended algebra.

\section{Free relativistic particle with fractional spin}

We consider first a simple model of a relativistic particle in $2+1$ dimensions, described by the action

$$
I=\int L d \tau
$$

where

$$
L=\frac{m(\dot{x} \dot{n})}{\sqrt{\dot{n}^{2}}}
$$


is the Lagrangian, $\tau$ is an evolution parameter, $x^{\mu}$ are the coordinates of the particle, $\mu=0,1,2$, and the dot denotes the derivative with respect to $\tau$. The auxiliary vector $n^{\mu}$ is a space-like unit vector $\left(n^{2}=-1\right)$ which, as we shall see below, serves to describe the spin degree of freedom of the particle. We shall take the metric as $g_{\mu \nu}=\operatorname{diag}(1,-1,-1)$. Finally, $m$ is a parameter with dimension of mass. The constants $\hbar$ and $c$ are set to unity.

Let us consider now the Hamiltonian formulation of the system in order to show that our proposed Lagrangian (2) indeed describes a free relativistic particle of mass $m$ with arbitrary spin. With this aim we introduce the canonical momenta $p_{\mu}$ and $p_{\mu}^{(n)}$, conjugated to the generalized coordinate $x_{\mu}$ and $n_{\mu}$ respectively, and which satisfy the canonical Poisson brackets (PB):

$$
\begin{aligned}
& \left\{x_{\mu}, p_{\nu}\right\}=-g_{\mu \nu}, \quad\left\{n_{\mu}, p_{\nu}^{(n)}\right\}=-g_{\mu \nu} \\
& \left\{x_{\mu}, x_{\nu}\right\}=\left\{p_{\mu}, p_{\nu}\right\}=0, \quad\left\{n_{\mu}, n_{\nu}\right\}=\left\{p_{\mu}^{(n)}, p_{\nu}^{(n)}\right\}=0 .
\end{aligned}
$$

From the Lagrangian (2) we obtain the momenta:

$$
\begin{gathered}
p_{\mu}=\frac{\partial L}{\partial \dot{x}^{\mu}}=\frac{m \dot{n}_{\mu}}{\sqrt{\dot{n}^{2}}}, \\
p_{\mu}^{(n)}=\frac{\partial L}{\partial \dot{n}^{\mu}}=\frac{m}{\sqrt{\dot{n}^{2}}}\left(\dot{x}_{\mu}-\frac{(\dot{x} \dot{n}) \dot{n}_{\mu}}{\dot{n}^{2}}\right) .
\end{gathered}
$$

Eqs. (4) together with the condition $n \dot{n}=0$ lead to the primary constraints [13]

$$
\begin{gathered}
\Phi_{1}=p^{2}-m^{2}=0, \\
\varphi_{1}=(p n)=0 \\
\varphi_{2}=\left(p p^{(n)}\right)=0 .
\end{gathered}
$$

The mass-shell condition (5a) is a first-class constraint (i.e. its PB's with all the constraints of the system vanish), while (5b) and $(5 \mathrm{c})$ are second-class constraints since $\left\{\varphi_{1}, \varphi_{2}\right\}=p^{2}=m^{2} \neq 0$. It is also straightforward to prove that the canonical Hamiltonian $H_{c a n}=\dot{x} p+\dot{n} p^{(n)}-L$ is equal to zero and consequently, the total Hamiltonian of the system is a linear combination of the constraints (5),

$$
H=\Lambda_{1} \Phi_{1}+\lambda_{1} \varphi_{1}+\lambda_{2} \varphi_{2}
$$

where $\Lambda_{1}(\tau), \lambda_{1}(\tau), \lambda_{2}(\tau)$ are the Lagrange multipliers $\left(\Lambda_{i}\right.$ is associated with the first-class and $\lambda_{j}$ with the second-class constraints, hereafter). Let us introduce now the spin vector $S_{\mu}$ by analogy with the orbital angular momentum [9]. We shall define

$$
S_{\mu}=\varepsilon_{\mu \nu \lambda} n^{\nu} p^{(n) \lambda}
$$

where $\varepsilon_{\mu \nu \lambda}$ is the totally antisymmetric tensor $\left(\varepsilon_{012}=1\right)$.

From (7) it follows that

$$
\left\{S_{\mu}, S_{\nu}\right\}=\varepsilon_{\mu \nu \lambda} S^{\lambda}
$$


The quantity $M_{\mu \nu}=x_{\mu} p_{\nu}-x_{\nu} p_{\mu}+n_{\mu} p_{\nu}^{(n)}-n_{\nu} p_{\mu}^{(n)}$ is the conserved total angular momentum tensor. It is easy to show that eqs. (5b) and (5c) imply that $\varepsilon_{\mu \nu \lambda} S^{\nu} p^{\lambda}=$ 0 , i.e. the spin vector $S^{\mu}$ is parallel to the momentum $p_{\mu}$ on $2+1$ dimensions. Thus, we can write

$$
S^{\mu}=-\frac{\alpha p^{\mu}}{\sqrt{p^{2}}}
$$

where $\alpha$ is an arbitrary constant (in principle, it could be a function of $\tau$, but from the equations of motion (10) it follows that $\dot{\alpha}=0$ ), which fixes the spin of the particle. From the Hamiltonian (6) (or equivalently from the Lagrangian (2)) and the definition (7) we obtain the following equations of motion:

$$
\dot{p}_{\mu}=0, \quad \dot{S}_{\mu}=0,
$$

i.e. the model describes a free relativistic particle of mass $m$ and with any arbitrary spin due to the fact that the parameter $\alpha$ does not appear explicitly in the Lagrangian. We can pass now to the Dirac quantization [13] of the system described by the Hamiltonian (6) with the set of constraints (5). The second-class constraints (5b) and (5c) define the so-called Dirac brackets, which give the commutation rules:

$$
\begin{aligned}
& {\left[x_{\mu}, x_{\nu}\right]=-i \varepsilon_{\mu \nu \lambda} \frac{S^{\lambda}}{p^{2}},} \\
& {\left[x_{\mu}, p_{\nu}\right]=-i g_{\mu \nu},} \\
& {\left[p_{\mu}, p_{\nu}\right]=0,} \\
& {\left[n_{\mu}, n_{\nu}\right]=0,} \\
& {\left[p_{\mu}^{(n)}, p_{\nu}^{(n)}\right]=0,} \\
& {\left[n_{\nu}, p_{\nu}^{(n)}\right]=-i\left(g_{\mu \nu}+\frac{1}{p^{2}} p_{\mu} p_{\nu}\right) .}
\end{aligned}
$$

The first-class constraint (5a) turns into the equation specifying the physical quantum states of the system:

$$
\left(p^{2}-m^{2}\right) \psi=0
$$

Note also that the spin operator (7) commutes with the constraint (5a) and therefore, it is a physical observable of the theory.

Now we shall consider the model in which the spin of the particle is fixed (it enters explicitly as a parameter in the Lagrangian). With this aim, in addition to constraints (5), we introduce a new first-class constraint

$$
\Phi_{2}=S p+\alpha m=0
$$

where $S_{\mu}$ is given by eq.(7) $)^{\text {f }}$. The first-class constraints (5a) and (13) are the invariants (Casimir operators) of the Poincaré algebra in $2+1$ dimensions $[14,7]$. The requirement (5a) is the mass-shell condition and eq. (13) specifies the helicity, with

\footnotetext{
* A different model with more constraints than (5) and (13) was proposed in [9], leading to different Hamiltonian and Lagrangian. However, in that model $\ddot{x}_{\mu} \neq 0$. In our proposed model $\ddot{x}_{\mu}=0$ and consequently, $x_{\mu}$ describes the space-time coordinate of the free particle.
} 
$\alpha$ an (arbitrary) value of spin. Note also that the set of constraints (5), (13) leads to the relation $(9)$.

We take as the total Hamiltonian of the system the linear combination of the primary constraints (5) and (13)

$$
H=\Lambda_{1} \Phi_{1}+\Lambda_{2} \Phi_{2}+\lambda_{1} \varphi_{1}+\lambda_{2} \varphi_{2}
$$

(with $H_{\text {can }}=0$ ). Performing the inverse Legendre transformation, we find the Lagrangian

$$
L=m \sqrt{\frac{\left(\varepsilon_{\mu \nu \lambda} \dot{x}^{\mu} n^{\nu} \dot{n}^{\lambda}-\alpha \frac{\dot{n}^{2}}{m}\right)^{2}+(\dot{x} \dot{n})^{2}}{\dot{n}^{2}}} .
$$

By direct verification one can show that Lagrangian (15) leads to the set of constraints (5) and (13) as primary constraints and that no secondary constraints appear in the model. The corresponding canonical Hamiltonian is equal to zero.

In the Dirac quantization scheme, the first-class constraints (5a) and (13) define the equations for the physical quantum states of the system $[7,9]$

$$
\left(p^{2}-m^{2}\right) \psi=0, \quad(S p+\alpha m) \psi=0,
$$

while the second-class constraints (5b), (5c) give the commutation rules (11). Since under quantization no restrictions on the parameter $\alpha$ appear, the physical states described by the wave functions (16), are the states of a particle with mass $m$ and arbitrary spin $\alpha$. It is straightforward to verify that the equations of motions (10) are also satisfied in this case.

Thus we have shown that Lagrangians (2) and (15) describe a free relativistic particle with arbitrary spin, with the only difference that in the case of eq. (2), the spin of the particle is not fixed in the theory, while Lagrangian (15) depends explicitly on the value of $\operatorname{spin} \alpha$.

\section{Anyon in external electromagnetic field}

Let us study now the problem of introducing the interaction of electromagnetic field with a fractional spin particle. By analogy with the noninteracting case [7], where the one-particle states are specified by values assigned to the invariants (Casimir operators) of the Poincaré algebra, we shall define the single-anyon states in a constant electromagnetic field as the unitary representations of the relevant operator algebra in $2+1$ dimensions. For a uniform constant electromagnetic field, the corresponding extended algebra has the form:

$$
\begin{aligned}
{\left[S_{\mu}, S_{\nu}\right] } & =i \varepsilon_{\mu \nu \lambda} S^{\lambda}, \\
{\left[S_{\mu}, \pi_{\nu}\right] } & =i \varepsilon_{\mu \nu \lambda} \pi^{\lambda}, \\
{\left[S_{\mu}, \tilde{F}_{\nu}\right] } & =i \varepsilon_{\mu \nu \lambda} \tilde{F}^{\lambda}, \\
{\left[\pi_{\mu}, \pi_{\nu}\right] } & =i e \varepsilon_{\mu \nu \lambda} \tilde{F}^{\lambda}, \\
{\left[\pi_{\mu}, \tilde{F}_{\nu}\right] } & =0, \\
{\left[\tilde{F}_{\mu}, \tilde{F}_{\nu}\right] } & =0,
\end{aligned}
$$


where $\pi_{\mu}=p_{\mu}-e A_{\mu}, A_{\mu}=-\frac{1}{2} F_{\mu \nu} x^{\nu}$ is the vector potential, $\tilde{F}_{\lambda}=\frac{1}{2} \varepsilon_{\lambda \mu \nu} F^{\mu \nu}$ and $F_{\mu \nu}$ is the uniform constant electromagnetic field tensor, $F_{\mu \nu}=\partial_{\mu} A_{\nu}-\partial_{\nu} A_{\mu}$.

We can prove that the operator $\pi^{2}-2 e(\tilde{F} S)$ is the Casimir of the algebra (17). Therefore, in the classical theory we can take the quantity $\pi^{2}-2 e(\tilde{F} S)-m^{2}$ as the corresponding first-class constraint. We will suppose this constraint to be valid even for a nonuniform fieldf $\tilde{F}_{\mu}$ and replace the set of constraints (5) by the following one:

$$
\begin{gathered}
\Phi_{1}=\pi^{2}-2 e(\tilde{F} S)-m^{2}=0, \\
\varphi_{1}=(\pi n)=0, \\
\varphi_{2}=\left(\pi p^{(n)}\right)=0,
\end{gathered}
$$

where $S_{\mu}$ is defined as in (7). The second-class constraints (18b), (18c) imply that the spin vector $S_{\mu}$ is parallel to the momentum $\pi_{\mu}$ and we can write

$$
S_{\mu}=-\alpha \frac{\pi_{\mu}}{\sqrt{\pi^{2}}}
$$

Now we define the total Hamiltonian of the system as follows

$$
H=\Lambda_{1} \Phi_{1}+\lambda_{1} \varphi_{1}+\lambda_{2} \varphi_{2}
$$

where $H_{\text {can }}$ is taken to be equal zero; $\Phi_{1}, \varphi_{1}, \varphi_{2}$ are given by eqs.(18) and $\Lambda_{1}, \lambda_{1}, \lambda_{2}$ are the Lagrange multipliers. In this case, unlike the free one, we can not find the exact analytic expression for the Lagrangian, since the equations for the Lagrange multipliers $\Lambda_{1}$ and $\lambda_{1,2}$ become polynomials of high degrees. However, keeping only the terms up to linear approximation in the field strength $\tilde{F}_{\mu}$, we can perform the inverse Legendre transformation to obtain the following Lagrangian:

$$
L=\frac{m(\dot{x} \dot{n})}{\sqrt{\dot{n}^{2}}}\left(1+\frac{e}{m^{2}}(\tilde{F} S)\right)+e A \dot{x}
$$

where $S_{\mu}$ is defined in (7) with $p_{\mu}^{(n)}$ given by (4b). The equations of motion, which follow from (20) (or, equivalently (21)) read as

$$
\begin{gathered}
\dot{\pi}_{\mu}=\frac{e}{m} F_{\mu \nu} \pi^{\nu}+\frac{e}{m} S^{\nu} \partial_{\mu} \tilde{F}_{\nu}, \\
\dot{S}_{\mu}=\frac{e}{m} F_{\mu \nu} S^{\nu},
\end{gathered}
$$

which are the ones obtained in [12] by following a different approach. The first term in the r.h.s. of eq. (22a) is the Lorentz force, while the second one corresponds to the coupling of the dipole moment to the gradient of the field. Eq.(22b) is the Bargmann-Michel-Telegdi equation in $2+1$ dimensions for the precession of the spin in an external electromagnetic field [15]. From the latter equation it follows that the gyromagnetic ratio for anyons is $g=2$ (see also [11], [12]), a fact which is a direct consequence of the form of the Casimir operator of the algebra (17). Note also that

\footnotetext{
*This assumption leads to the correct equations of motions in a nonuniform external electromagnetic field (see eqs. (22)).

** In addition, this linear approximation in the field strength provides us with the possibility to compare our results with the ones obtained previously in the literature in the same approximation.
} 
we can come to the same conclusion from the system of constraints (18). Indeed, if one takes $\Phi_{1}$ in the form $\Phi_{1}=\pi^{2}-2 e \eta(\tilde{F} S)-m^{2}=0$, with $\eta$ a constant, it turns out that $\Phi_{1}$ is a first-class constraint if and only if $\eta=1$. Thus, $g=2$ due to the parallelness [12] of spin and momentum in $2+1$ dimensions (guaranteed in our case by the second-class constraints $(18 b),(18 c))$ and due to the fact that $\Phi_{1}$ should be a first-class constraint.

Let us remark that the equations of motion are obtained in the form (22) since on the mass-shell (i.e. when the equations of motion are taken into account) $\dot{n}_{\mu}$ is parallel to $\dot{x}_{\mu}$.

It is interesting to notice that if we identify the time-like vector $\dot{n}_{\mu}$ with $\dot{x}_{\mu}$ by setting $\dot{n}_{\mu}=-\dot{x}_{\mu}$, the Lagrangian (21) coincides with the standard Lagrangian (in this case in any dimension), for a spinless charged particle in an electromagnetic field

$$
L=-m \sqrt{\dot{x}^{2}}+e A \dot{x} .
$$

Let us consider now the quantization of the system, following the Dirac method [13]. The second-class constraints (18b), (18c) can be used to obtain up to terms linear in $\tilde{F}_{\mu}$ the following commutation rules:

$$
\begin{aligned}
& {\left[x_{\mu}, x_{\nu}\right]=-i \varepsilon_{\mu \nu \lambda} \frac{S^{\lambda}}{\pi^{2}}\left(1-e \frac{(\tilde{F} S)}{\pi^{2}}\right)} \\
& {\left[x_{\mu}, \pi_{\nu}\right]=-i g_{\mu \nu}\left(1-e \frac{(\tilde{F} S)}{\pi^{2}}\right)+i e \frac{\tilde{F}_{\mu} S_{\nu}}{\pi^{2}}} \\
& {\left[\pi_{\mu}, \pi_{\nu}\right]=i e \varepsilon_{\mu \nu \lambda} \tilde{F}^{\lambda}=i e F_{\mu \nu},} \\
& {\left[n_{\mu}, n_{\nu}\right]=0} \\
& {\left[p_{\mu}^{(n)}, p_{\nu}^{(n)}\right]=0,} \\
& {\left[n_{\mu}, p_{\nu}^{(n)}\right]=-i g_{\mu \nu}-i \frac{\pi_{\mu} \pi_{\nu}}{\pi^{2}}\left(1-e \frac{(\tilde{F} S)}{\pi^{2}}\right)}
\end{aligned}
$$

The first-class constraint (18a) is imposed as an operator on the physical quantum states giving the equation:

$$
\left(\pi^{2}-2 e(\tilde{F} S)-m^{2}\right) \psi=0
$$

This equation was heuristically assumed in [10] for an anyon in a constant magnetic field in the context of relativistic fractional quantum Hall effect and recently it was obtained in [12] by introducing the minimal coupling with the electromagnetic field in the sympletic structure. As it was shown in [12], the nonrelativistic limit of eq. (24) gives for the magnetic moment of the anyon $\mu=-\frac{e \alpha}{m}$ and we see again that $g=2$.

It might be interesting to mention here that, in the linear approximation in the field $\tilde{F}_{\mu}$, the Lagrangian (21) can be obtained from the free Lagrangian (2) by the following substitution:

$$
\begin{aligned}
& \dot{x}_{\mu} \rightarrow \dot{x}_{\mu}+\frac{e}{m} \frac{(\dot{x} \dot{n})}{\sqrt{\dot{n}^{2}}} A_{\mu}, \\
& \dot{n}_{\mu} \rightarrow \dot{n}_{\mu}+\frac{e}{m} \sqrt{\dot{n}^{2}} A_{\mu}-\frac{e}{m} \frac{(\dot{x} \dot{n})}{\sqrt{\dot{n}^{2}}} F_{\mu \nu} n^{\nu} .
\end{aligned}
$$


Finally, some remarks are in order concerning the possible extension of the model described by Lagrangian (15) to the case with electromagnetic interaction. The introduction of a new constraint $\Phi_{2}=S \pi+\alpha m=0$ together with eqs. (18) will lead to a contradictory system of constraints ${ }^{*}$ and besides, as we have seen before, the algebra (17) has only one Casimir operator, namely, $\Phi_{1}$ given in (18a). In order to obtain a consistent theory, where the spin parameter $\alpha$ appears explicitly in the Lagrangian, we are forced to modify the set of constraints. This question is under study.

We can also treat the electromagnetic field as a dynamical variable by adding to the Lagrangian (21) the usual term $-\frac{1}{4} F_{\mu \nu} F^{\mu \nu}$ and construct the quantum electrodynamics for anyons. Another interesting problem is to formulate the quantum version of the theory in terms of path integrals.

\section{Acknowledgements}

This work started in collaboration with Alejandro Cabo and Hugo Perez Rojas. We are grateful to them for many important discussions and comments at an early stage of the work. It is our pleasure to thank I. Komarov and C. Montonen for several clarifying discussions. One of us (R.G.F.) would like to thank the ICSCWorld Laboratory for financial support. D.L.M. would like to thank the Centre for International Mobility (CIMO), Finland, for providing with financial support.

${ }^{*)}$ The new constraint $\Phi_{2}=S \pi+\alpha m=0$ can not be first-class, since not all its PB's with the other constraints (18), namely with (18b) and (18c), vanish. Considering it as second-class, would form together with (18b) and (18c) a total odd number (three) of second-class constraints which is not possible, unless one adds another second-class constraint. 


\section{References}

[1] J.M. Leinaas and J. Myrheim, Nuovo Cim. B37 (1977) 1;

F. Wilczek, Phys. Rev. Lett. 48 (1982) 1144; ibid. 49 (1982) 957. For a recent review, see F. Wilczek, Fractional Statistics and Anyon Superconductivity (World Scientific, Singapore, 1990).

[2] F. Wilczek and A. Zee, Phys. Rev. Lett. 51 (1983) 2250;

Y.S. Wu and A. Zee, Phys. Lett. B147 (1984) 325;

M.J. Bowick, D. Karabali and L.C.R. Wijewardhana, Nucl. Phys. B271 (1986) 417.

[3] I. Dzyaloshinskii, A. Polyakov and P. Wiegmann, Phys. Lett. A127 (1988) 112;

P.K. Panigrahi, S. Roy and W. Scherer, Phys. Rev. Lett. 61 (1988) 2827.

[4] K. Paul and A. Khare, Phys. Lett. B174 (1986) 420;

H.J. de Vega and F.A. Schaposnic, Phys. Rev. Lett. 56 (1986) 2654; J. Fröhlich and P. Marchetti, Commun. Math. Phys. 121 (1989) 177.

[5] J. Hong, Y. Kim and P.Y. Pac, Phys. Rev. Lett. 64 (1990) 2230;

R. Jackiw and E.J. Weinberg, Phys. Rev. Lett. 64 (1990) 2234.

[6] C. Hagen, Phys. Rev. D31 (1985) 2135; T.H. Hansson, M. Roček, I. Zahed and S.C. Zang, Phys. Lett. B214 (1988) 475; G.W. Semenoff, Phys. Rev. Lett. 61 (1988) 517; S. Deser and A.N. Redlich, Phys. Rev. Lett. 61 (1988) 1541; P. Gerbert, Int. J. Mod. Phys. A6 (1991) 173.

[7] R. Jackiw and V.P. Nair, Phys. Rev. D43 (1991) 1933.

[8] M.S. Plyushchay, Phys. Lett. B262 (1991) 71; Nucl. Phys. B362 (1991) 54.

[9] M.S. Plyushchay, Int. J. of Mod. Phys. A6 (1992) 7045.

[10] A. Cabo, M. Chaichian, R. Gonzalez Felipe, A. Perez Martinez and H. Perez Rojas, Phys. Lett. A166 (1992) 153.

[11] I.I. Kogan and S.W. Semenoff, Nucl. Phys. B368 (1992) 718.

[12] C. Chou, V.P. Nair and A.P. Polychronakos, Phys. Lett. B304 (1993) 105.

[13] P.A.M. Dirac, Lectures on Quantum Mechanics (Belfer Graduate School of Science, Yeshira University, New York, 1964).

[14] B. Binegar, J. Math. Phys. 23 (1982) 1511.

[15] See for example, C. Itzykson and J-B. Zuber, Quantum field theory (McGraw Hill, New York, 1980). 